\title{
Shop-Window Lighting in the Mediterranean Countries: The Use of Sun to Improve Visual Appeal and Reduce Energy Demand
}

\author{
By Elena Anagnou* \\ Helena Coch $^{\dagger}$ \\ Isabel Crespo
}

The present study deals with the potential reduction of energy consumption for lighting of shop window displays in the Mediterranean countries. Urban commerce has a very high impact on economics and it is a highly energy-consuming sector. Light has the power of attracting people's attention, which is one of the goals of the trading and selling activity and very high illuminance levels are usually recommended. In the Mediterranean areas, where daytime lasts for many hours, commercial activity takes place mainly under sunshine conditions and shop windows frequently fail to fulfil their main corporative goal, namely the unobstructed observation of the products exhibited. The necessary increase of artificial lighting illuminance levels to accent interior light conditions, due to extremely high-luminance urban surroundings, leads to an important increase of energy consumption. Nevertheless, the results are generally very poor, due to reflections and other kinds of visual problems. The present study evaluates the visual and energetic benefits of an innovative passive design that obstructs solar rays and redirects them into the interior of the shop window scene. A scale model of this new design confirms the visual benefits produced by its use, via the different luminance maps tested.

Keywords: display lighting, passive design, saving energy, shop-window.

\section{Introduction}

The image of the Mediterranean cities, where climate favours exterior human activities, is conserably affected by urban commerce and storefronts. Being a fundamental element of the commercial process, a proper visual presentation of shop windows is crucial. Lighting contributes to the creation of a corporate identity, ideally, it assists the production of the image desired by the trader and together with other components of the design it contributes to sales promotion (Rea, 2000). The present study analyzes the visual inconveniences of window displays associated with the Mediterranean climate and how they are or should be dealt with, so that the aesthetic and commercial function of shop windows will not be inhibited.

\footnotetext{
${ }^{*}$ Researcher, Polytechnic University of Catalonia, Spain.

$\dagger$ Polytechnic University of Catalonia, Spain.

* Polytechnic University of Catalonia, Spain.
} 


\section{In the Mediterranean Cities, the Shopping is Combined with Promenade under the Sun}

In Mediterranean climates, the temperature favours urban commerce, since duration of daily solar radiation is extensive and solar intensity is prevalent throughout the whole year. For example, in one of the most commercial districts of Barcelona, Eixample, the urban scenery offers optimal conditions regarding the use of the mild climate of Barcelona; at pedestrian level the broad avenues are offered to enjoy the sun and good weather and combine promenade with shopping (see figure 1).

Figure 1. In Mediterranean Cities like Barcelona, Urban Fabric and Mild Climate Favour Urban Commerce

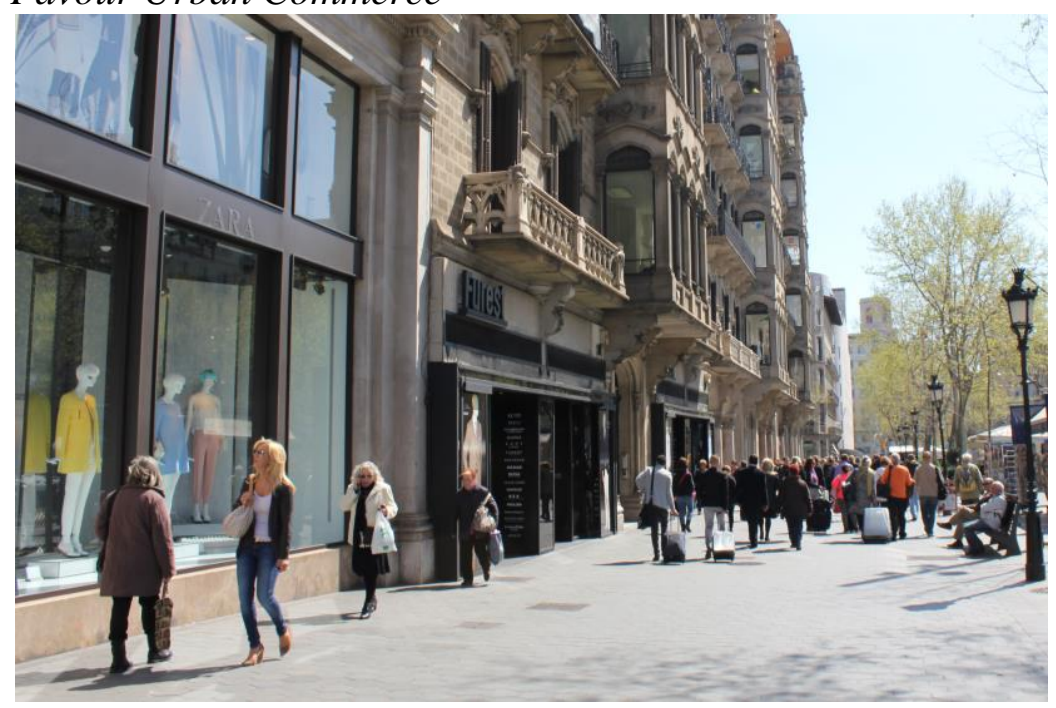

However, although in the Mediterranean countries shop-window glazing mainly takes place under sunlight, shop-window light scenes are basically designed for their night presentation. This fact has significant inconveniences for the shop-window glazing procedure that derive from the exposure of window displays to varying light conditions in the outside environment; due to the very high level of exterior illuminance, the visual goals of window displays are often impeded.

\section{High Energy Consumption yet Poor Visual Outcome}

The lighting design of window displays is required to ensure visual comfort and good vision of the products exhibited (Anagnou and Coch 2012). In order to do so, adequate illuminance levels and luminance contrasts must be secured. A recommended value for luminance ratio between the illuminated object and its surrounding area is 3:1 (Carillo et al. 2010). Regarding illuminance values, certain studies prove that a higher illuminance level implies that more passers-by are attracted by the shop windows (Carillo et al. 2010). As a result, the consumption of energy for the lighting of window displays is rather high (Freyssinier et al. 2006). In Catalonia, window display 
lighting constitutes $11.2 \%$ of the total energy consumption for the lighting of a typical store (Institut Catalá d’Energia, 2008).

However, despite the high energy consumption, visual inconveniences insist; during the research discussed in this paper, a study of a representative sample of shop windows of the city of Barcelona has been performed analyzing their visual results. The basic tools of this analysis have been the luminance maps and evaluation, via computer application, of the odds of glare. The study has confirmed and identified the main inconveniences that can impair the visual result and, therefore, the commercial purposes, during daytime hours. Specifically, the most frequent problems are: failure to reach the appropriate contrasts for the projection of exhibits, glare and annoying reflections.

Indeed among the analysed stores, the visual presentation of $65 \%$ of them failed to reach a contrast of 3:1 between all exhibits and their surroundings, as well as $65 \%$ of them failed to avoid annoying reflections (see Figure 2). In addition, the conducted analysis confirms that the commercial storefronts mostly affected by such inconveniences are those receiving direct solar incidence, weather in the actual presentation of the window or in immediately adjacent areas.

In order to help face these visual disadvantages resulting from very high levels of exterior illuminance, a remedy often applied is to increase artificial illuminance projected during the day, in contrast to the one projected overnight. However, the intensity of sunlight in the Mediterranean countries is so high that artificial lighting cannot compete with the natural one making it vain for any power to increase during the day, along with the corresponding energy additional charge this practice entails.

This study instead evaluates the visual and energetic benefits of an innovative passive design. 
Figure 2. (a) Graphic Showing Percentage of Commerce Failing to Reach Contrast 3:1 Between All Exhibits and their Surroundings, Plus An Example (B) Graphic Showing Percentage of Commerce with Presence of Annoying Reflections, Plus An Example

Contrast $<3: 1$

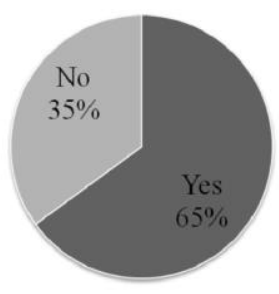

a)

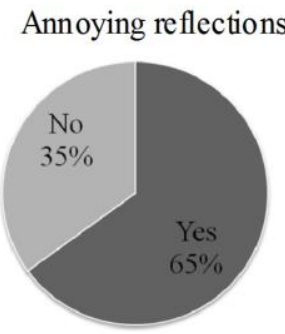

b)
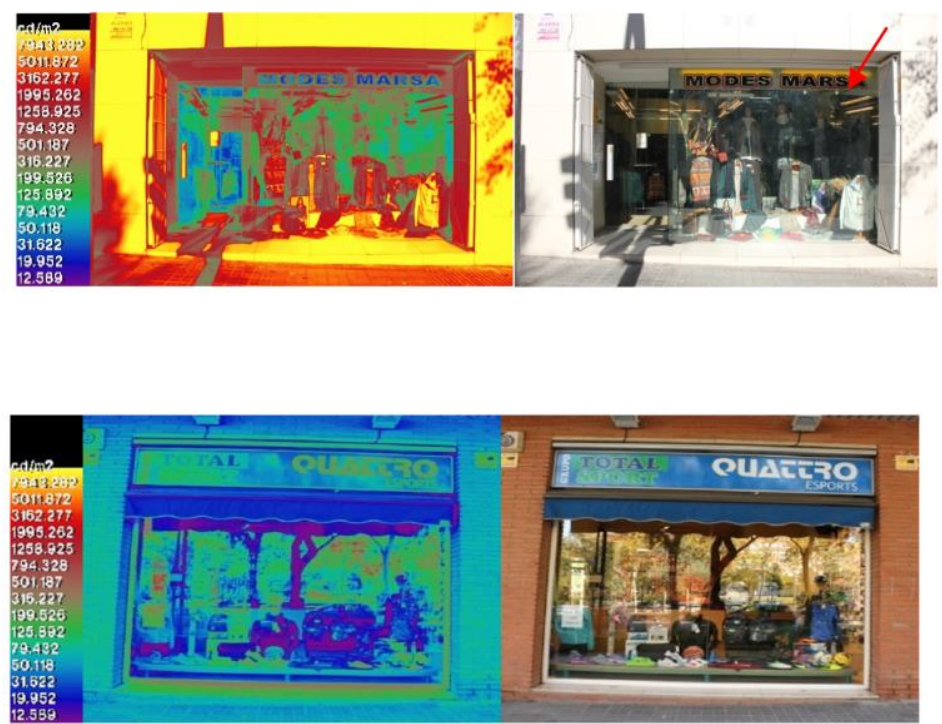

\section{Methodology}

The present research evaluates the visual and energetic benefits of a passive design that obstructs solar rays and redirects them into the interior of the shop window scene. In fact, the improvement of the quality of the observation of exhibits during daytime is assessed, along with the possible reduction in energy consumption.

\section{Selection of the Case}

The center of Barcelona, Eixample, has been considered ideal for this study because it is a district with a significant concentration of commercial activity and it has a structure that favours sun-lighting of its wide streets and building facades; in addition, the regulated matrix of its urban fabric facilitates such a study and allows the generalization of results across its surface (see Figure 3). 
Figure 3. The Regulated Matrix of Eixample District

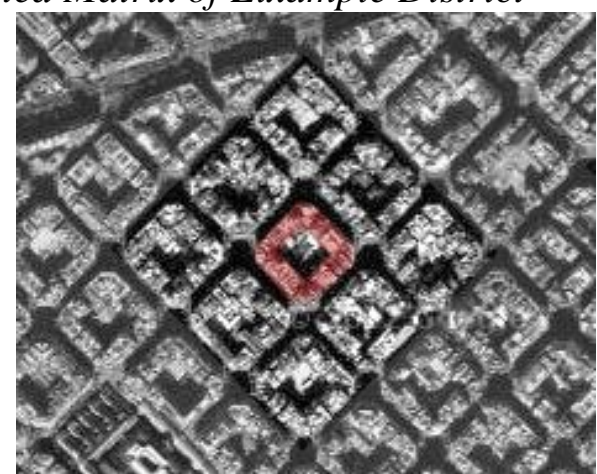

Source: https://www.google.com/maps/preview (subsequently elaborated image)

A segment of the Eixample has been modelled in a simulation program that incorporates solar trajectories (Heliodon). After analyzing the data obtained during the study, concerning the time of exposure to solar radiation and the percentage of illuminated surface is observed as expected, that: a) The facades of the ground floor facing south are the most affected. b) The most detrimental period, when higher percentage of the surfaces of the ground floor is exposed to sunlight despite the obstruction of the urban fabric, is summer (see Figure 4). Furthermore, it is the period with the highest average monthly hours of sunshine in Barcelona.

Figure 4. a) Design and Emissivity Percentage Isochronous of South Facades. b) Percentage of Illuminated Surface, Evolution throughout the Year
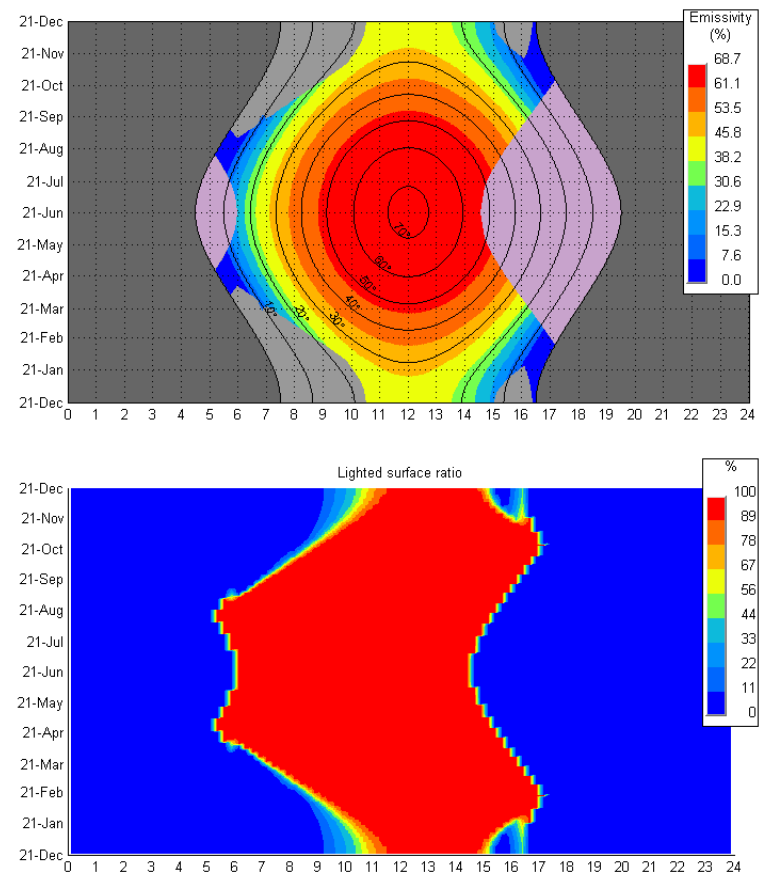


\section{Development of the Passive Design Component}

A typical window display of Barcelona has been selected among the sample observed, referred in introduction, that is about four meters high, one meter wide and facing south. Based on the solar diagram of the city, several design tests have been performed in order that, when a system of reflective surfaces is incorporated in a typical window facing south, it could make the most of the solar incidence during the most unfavourable conditions; the latter being summer solstice, as confirmed with Heliodon.

The design that is finally proposed here (see Figure 5), is based on two successive light reflections. Rays that fall upon the first and curved surface will be reflected symmetrically around the axis joining the point of incidence and its centre of curvature (Peoglos et al. 2004); subsequently, they will be cast on the plane surface and will be reflected towards the interior of the scene.

Figure 5. (a) Geometry of the Component Proposed (b) Conduction of Natural Light in the Interior of the Scene

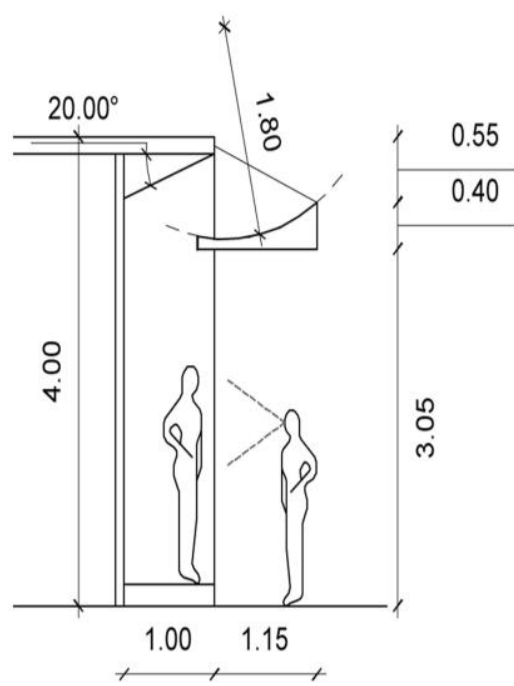

a)

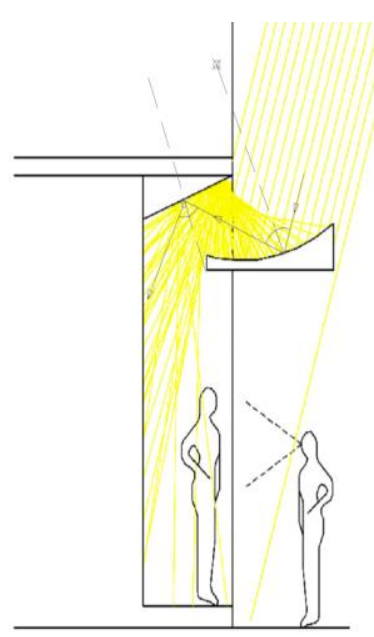

b)

Development of the Scale Models

Two scale models of the window analyzed, the one being independent of and the other one based on the proposed system, have been developed. As in the present study the effect of chromatic contrast is not analyzed, all the components of the scene have been chosen to be of soft and slightly contrasting colours (see Figure 6). 
Figure 6. Model Based on the System Proposed

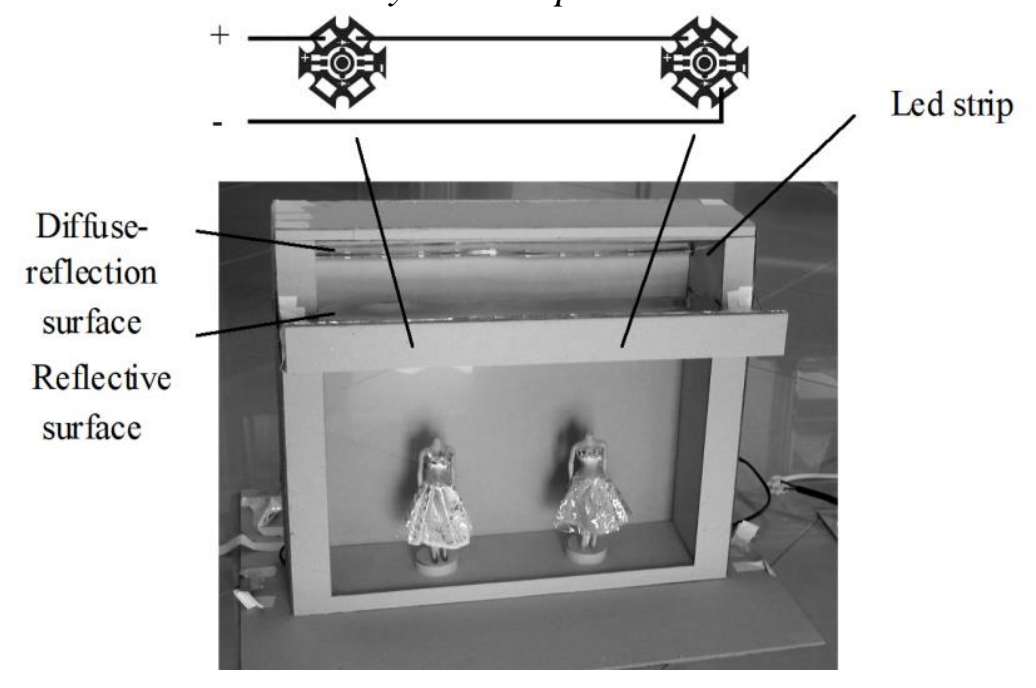

The dimensions of the models have been adjusted to the artificial lighting projected, giving special care so that both the area of the surfaces and the luminous flux they receive, would be correctly scaled. In each one of the models two LED sources have been placed above two mannequins and $30 \mathrm{~cm}$ of led stripe above the background of the scene ${ }^{1}$. In order to simulate the illuminance of the real window display, after measuring the luminous intensity of both real and model light sources, and applying the basic laws of photometry, the appropriate scale of the model has been defined to $\mathrm{dM}=0,09 \mathrm{dR}$ ${ }^{2}$. A potentiometer has also been connected to the led stripe, so that its intensity could be dimmed and, in this manner, possible reduction of energy for vertical illumination could be evaluated.

\section{Simulation of the Desired Exterior Light Conditions}

The study took place in Athens on December 10, during the time of zenith. In order to simulate the summer solstice in Barcelona ( $72^{\circ}$ during the zenith) the table where each model was placed has been rotated through $43^{\circ}$ (see Figure 7) equal to the difference of solar altitude on December 10 in Athens (29o during the zenith). Furthermore, in order to obtain an overall assessment of the results for the entire year, a simulation of the equinox and winter solstice in Barcelona ( $49^{\circ}$ and $26^{\circ}$ correspondingly during the zenith) were simulated rotating the table through $20^{\circ}$, and $-3^{\circ}$ correspondingly.

\footnotetext{
${ }^{1}$ A Prolight PM2B 3LxS-SD-3W above each mannequin and a power LED a strip led of 40W type 3528 for vertical illumination.

${ }^{2} \mathrm{~d}_{\mathrm{M}}=$ dimensions of the model, $\mathrm{d}_{\mathrm{R}}=$ dimensions of the real window display
} 
Figure 7. Simulation of the Desired Exterior Lighting Conditions by Rotating Through $43^{\circ}$ the Table Where the Model Is Placed
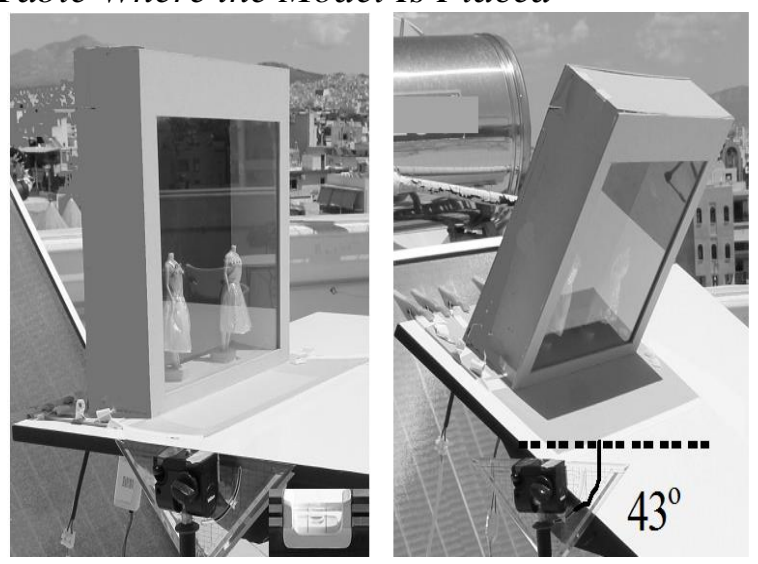

Evaluation of Visual Differentiation, Depending On the Existence of the Proposed System

The comparison of the visual outcome of the two different models has been realized via the observation of photographs and their equivalent luminance maps, in addition to the observation in situ. The comparison took into account the following visual inconveniences:

1. Annoying reflections.

2. Achievement of 3:1 contrast between products and their surrounding area.

3. Glare.

In order to compare the possibility of glare in every situation, the Radiance based tool, called Evalglare, which evaluates that possibility through the daylight glare probability index, DGP (Wienold 2009) has been used in addition to the luminance maps.

\section{Results and Discussion}

The comparison of the visual result of the two scenes, the one independently of and the other one based on the proposed design, has resulted the expected improvement. Via the observation of the photographs and the luminance maps derived from them, the positive effect of the redirection of the sunlight into the scene is appreciated (see Figure 8). 
Figure 8. (a) Photograph and Luminance Map (b) DGP Index. Comparison of the Two Models, without (left) and with (right) the System Proposed

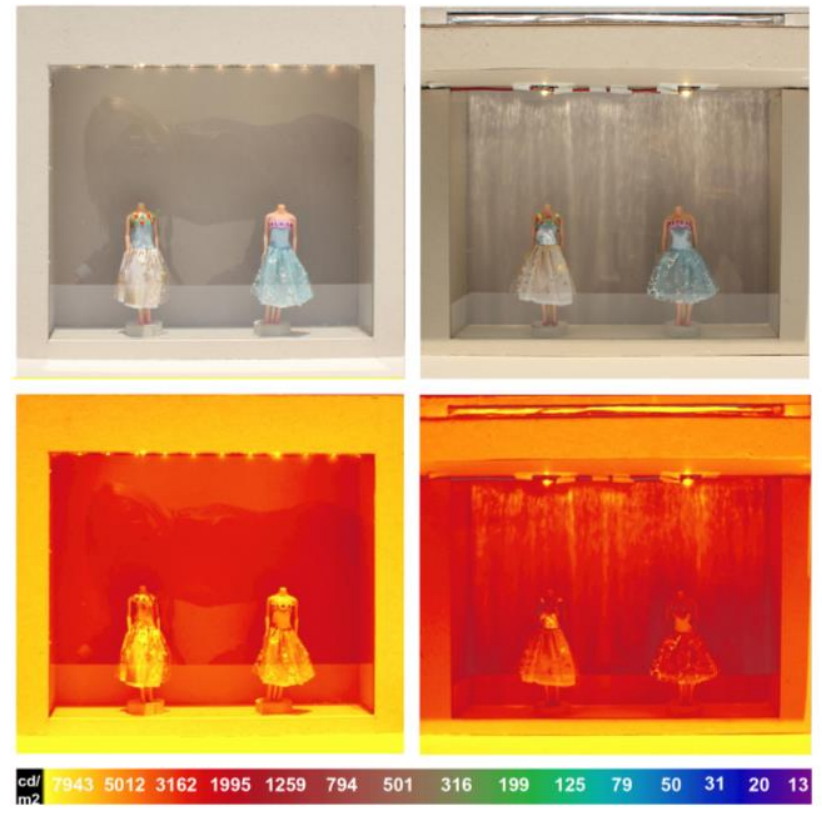

a)
Daylight glare probability index in the two models, without and with the system proposed correspondingly

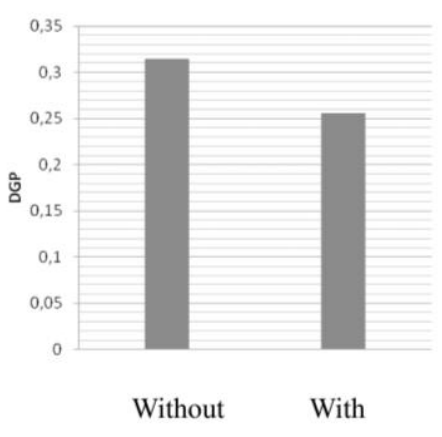

b)

Specifically, regarding the model of the proposed system, the following parameters are observed:

1. The reflections on the glass of the scene are significantly moderated compared to the model without the system.

2. The desired contrast of 3:1 between the products and their surrounding area is achieved, avoiding thought high contrasts dued to solar incidence on them.

3. Less areas of high luminance that can dazzle are generated comparing to the model without the system. The comparison of DGP index for the two cases is indicative.

Concerning the rest of the seasons, the design proposed has no negative effect. On the contrary, the effect against the inconveniences of glare and annoying reflexions are positive throughout the year.

Following, in order to evaluate the possibility of energy reduction, the power of the background lighting of the scene has been decreased in the case of the design proposed; applying the 1/3 of the illuminance projected before, the result has been again positive (see Figure 9). Even with a drastic reduction of power applied when the proposed design is incorporated, regarding the model of the proposed system, the following parameters are observed:

1. The 3:1 contrast is once again achieved.

2. The generated reflections are significantly lighter. 
3. Probability of glare is smaller.

All these effects were expected, since solar light intensity that is introduced in the scene is the one increasing significantly the illuminance in it; not artificial lighting.

Figure 9. (a) Photograph and Luminance Map (b) DGP index. Comparison of the Two Models, without (left) and with (right) the System Proposed When In Addition Wall Wash Lighting Is Reduced to 1/3

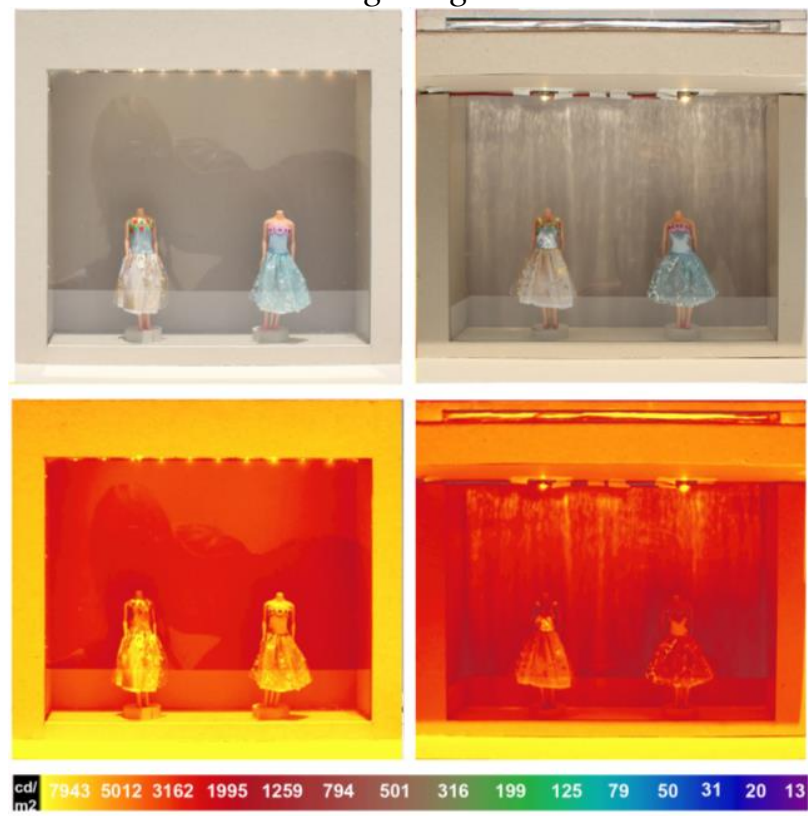

a)
Daylight glare probability index in the two models, without and with the system proposed correspondingly

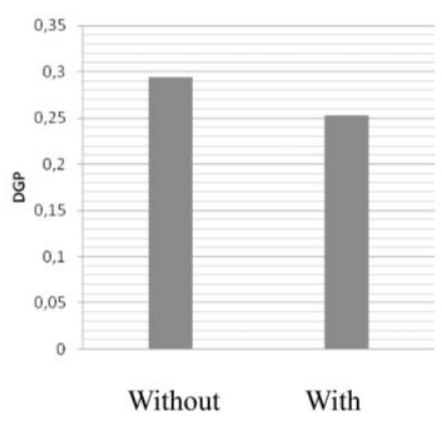

b)

\section{Conclusion}

This paper results in a passive design proposal that can offer very positive effects on the visual result of shop windows, improving the quality of their observation, thus fulfilling their commercial visual goals during the day and, in addition, reducing energy consumption. In the Mediterranean countries, the inability of artificial lighting to compete with high solar illuminance makes it vain for any power to increase during daytime, along with the corresponding energy charge this practice entails. Instead, the increase of interior illuminance levels of shop windows by using the very illuminance of the sun may improve the visual outcome and reduce energy consumption for the lighting of the window display.

The positioning of the proposed design of sunlight redirection, with the dual effect of both obstruction of its incidence and the benefit of its intensity to increase illuminance in the interior of the shop window, has very positive effects on its visual presentation, especially in terms of reducing annoying reflexions and the possibility of glare. Moreover, these effects are valid even 
when reducing the installed power for the lighting of the background of the scene, thus making possible, in combination with the use of sensors and resistors, further reduction of energy consumption for the lighting of the display window. This new lighting passive system results in a very simple, effective and low cost solution that can be easily applied in existing shopwindows, with considerable visual and environmental benefits when incorporated in the highly energy-consuming display windows.

\section{References}

Anagnou E, Coch H (2012) Escaparates de Barcelona: El impacto del alumbrado comercial exterior a la ciudad [Barcelona windows: The impact of outside commercial lighting to the city]. Luces Cei, Febrero 45: 18-24.

Carillo C, Diaz-Dorado E, Cidrás J, Bouza-Pregal A, Falcón P, Fernández A, ÁlvarezSánchez A (2013) Lighting control system based on digital camera for energy saving in shop windows. Energy and Buildings 59: 143-151

Freyssinier JP, Frering D, Taylor J, Narendran N, Rizzo P (2006) Reducing light energy use in retail display windows. Sixth International Conference on Solid State Lighting. San Diego: Proceeding of SPIE.

Institut Catalá d’Energia (2008) Caracterització del consum energétic en els establiments del sector de pimes de comerç $i$ serveis de Catalunya [Characterization of energy consumption on the premises of the SME sector of trade and services of Catalonia]. Barcelona.

Peoglos B, Raptis I, Christodoulides C (2004) Optical Instruments. In B Peoglos, I Raptis, C Christodoulides, Experimental Physics Techniques. Athens: National Technical University of Athens 3: 39-82.

Rea M (2000) IESNA Lighting Handbook: Reference \& application. 9th Edition. New York: Illumination Engineering Society of North America.

Wienold J (2009) Dynamic daylight glare evaluation. IBPSA Conference. Glasgow: Building Simulation: 944-951. 
